\section{Early Bilateral Amniotic Membrane Transplantation in the Management of Severe Ocular Involvement from Acute Toxic Epidermal Necrolysis in a Chinese Pediatric Patient}

\author{
Kendrick Co Shih ${ }^{1,2}$, Suk Ming Yim², Johnny Chun Yin Chan ${ }^{3}$, Shun Kit Chan ${ }^{2}$, Jimmy Shiu Ming Lai ${ }^{1,2}$ and Leonard Yuen ${ }^{2^{*}}$ \\ ${ }^{1}$ Department of Ophthalmology, LKS Faculty of Medicine, University of Hong Kong, Pokfulam, Hong Kong, China \\ ${ }^{2}$ Department of Ophthalmology, Queen Mary Hospital, Pokfulam, Hong Kong, China \\ ${ }^{3}$ Dermatology Division, Department of Medicine, LKS Faculty of Medicine, University of Hong Kong, Pokfulam, Hong Kong, China
}

*Corresponding author: Dr. Leonard Yuen MPH, MRCOphth, FRCSEd, FHKCOphth, FHKAM, Department of Ophthalmology, Queen Mary Hospital, Pokfulam, Hong Kong, China, Tel: (+852) 2255 3612; Fax (+852) 2255 5818; E-mail: lyuen@post.harvard.edu

Received date: August 27, 2014, Accepted date: September 24, 2014, Published date: September 30, 2014

Copyright: $\odot 2014$ Shih KC, et al. This is an open-access article distributed under the terms of the Creative Commons Attribution License, which permits unrestricted use, distribution, and reproduction in any medium, provided the original author and source are credited.

\begin{abstract}
Introduction: Toxic Epidermal Necrolysis (TEN) is a rare but potentially life-threatening muco-cutaneous condition associated with idiosyncratic hypersensitivity to certain drugs. Ophthalmic involvement is common, typically affecting the ocular surface and eyelids. Survivors often suffer from resulting bilateral blindness and ocular dryness or pain.
\end{abstract}

Objective: To report the successful management of severe ocular surface disease during the acute stage of toxic epidermal necrolysis using early amniotic membrane transplantation on both eyes in a Chinese paediatric patient.

Design: Interventional case report

Case report: A 15 year-old Chinese girl was transferred to the intensive care unit of Queen Mary Hospital, Hong Kong with TEN after taking oral cefuroxime and diclofenac. She developed bilateral keratoconjunctivitis, diffuse corneal epithelial defects (80-90\% of cornea surface) and later bilateral symblephara. After initial treatment with daily rodding, topical lubricants, steroids and antibiotics, there was no improvement in her condition. Bilateral amniotic membrane transplantation (AMT) was performed over the cornea, fornix, tarsal and bulbar conjunctiva on day 10 of illness.

On discharge from the hospital (post-operative week 7), the patient had pinhole visual acuity of 6/7.5 in the right eye and $6 / 6$ the left eye. She was eventually weaned off all topical medication Visual acuity eventually recovered to $6 / 6$ in both eyes by week 20 after surgery. There was mild residual forniceal symblepharon and eyelid margin keratinization. She continues to require regular lubricants for her chronic ocular surface condition.

Conclusion: Early use of AMT minimized ocular damage and prevented severe vision loss in a Chinese pediatric patient with severe bilateral ocular surface inflammation secondary to TEN. Effective management is dependent on early and active communication between the patient's family, pediatricians, intensive care physicians and ophthalmologists.

Keywords: Early amniotic membrane transplantation; Toxic epidermal necrolysis; Acute ocular surface inflammation; Chinese; Paediatric patient

\section{Abbreviations}

TEN: Toxic Epidermal Necrolysis; SJS: Steven-Johnson Syndrome; AMT: Amniotic Membrane Transplantation; BSCL: Bandage Soft Contact Lens

\section{Introduction}

Toxic Epidermal Necrolysis (TEN) is a rare but potentially life threatening muco-cutaneous condition first described by Lyell in 1958 [1]. Together with Steven-Johnson Syndrome (SJS), they form opposite ends of a spectrum of acute exfoliating diseases of the skin and mucous membranes due to a full-thickness epidermal necrosis. Differentiation between the two conditions is dependent on the extent of total body surface area involved and has important prognostic implications. TEN represents the most extensive form of the disease and a diagnosis is made when more than $30 \%$ of total body surface area is involved [2].

Ophthalmic involvement is common in patients with TEN, with more than $50 \%$ of patients developing acute ocular surface inflammation [3]. Even with aggressive use of lubrication and topical steroids, visual prognosis is often poor as a result of margin deformation, conjunctival scarring, symblephera formation, limbal stem cell deficiency, dry eyes and recurrent or persistent epithelial defects. 
Citation: Shih KC, Yim SM, Chan JCY, Chan SK, Lai JSM, et al. (2014) Early Bilateral Amniotic Membrane Transplantation in the Management of Severe Ocular Involvement from Acute Toxic Epidermal Necrolysis in a Chinese Pediatric Patient. J Clin Exp Ophthalmol 5: 358. doi: $10.4172 / 2155-9570.1000358$

Page 2 of 6

Over the past decade, the introduction of amniotic membrane transplantation in ocular surface reconstruction after SJS/TEN has greatly aided in the rehabilitation and supportive management of chronic ocular surface disease [4]. More recently, AMT has been performed in the acute inflammatory phase with the aim of preventing long-term ocular surface complications. The evidence for this approach has so far been very encouraging [5-13].

Here we report the ocular and visual outcomes of a Chinese pediatric patient who underwent early amniotic membrane transplantation (AMT) during the acute inflammatory phase of TEN.

\section{Case Report}

A 15 year-old Chinese girl was admitted to Kwong Wah Hospital (KWH), Hong Kong, for severe oral blistering, lip swelling, bilateral conjunctivitis, dermal sloughing on $2^{\text {nd }}$ July 2012. Three months prior, she started on an unknown regime of Traditional Chinese Medicine for irregular menses, and immediately before admission, the patient took oral cefuroxime and diclofenac for an upper respiratory tract infection for two days. On admission the patient was initially diagnosed with Stevens-Johnson's Syndrome (SJS) with 10\% skin involvement. She was started on high dose intravenous methylprednisolone and intravenous immunoglobulin. Despite the measures, her skin involvement reached $>30 \%$ by the $5^{\text {th }}$ of July and was now complicated by respiratory failure and thrombocytopenia. The diagnosis was subsequently labelled as toxic epidermal necroylsis (TEN). She was intubated under sedation and transferred to the pediatric intensive care unit, Queen Mary Hospital, Hong Kong for continued care on the same day (Figure 1).

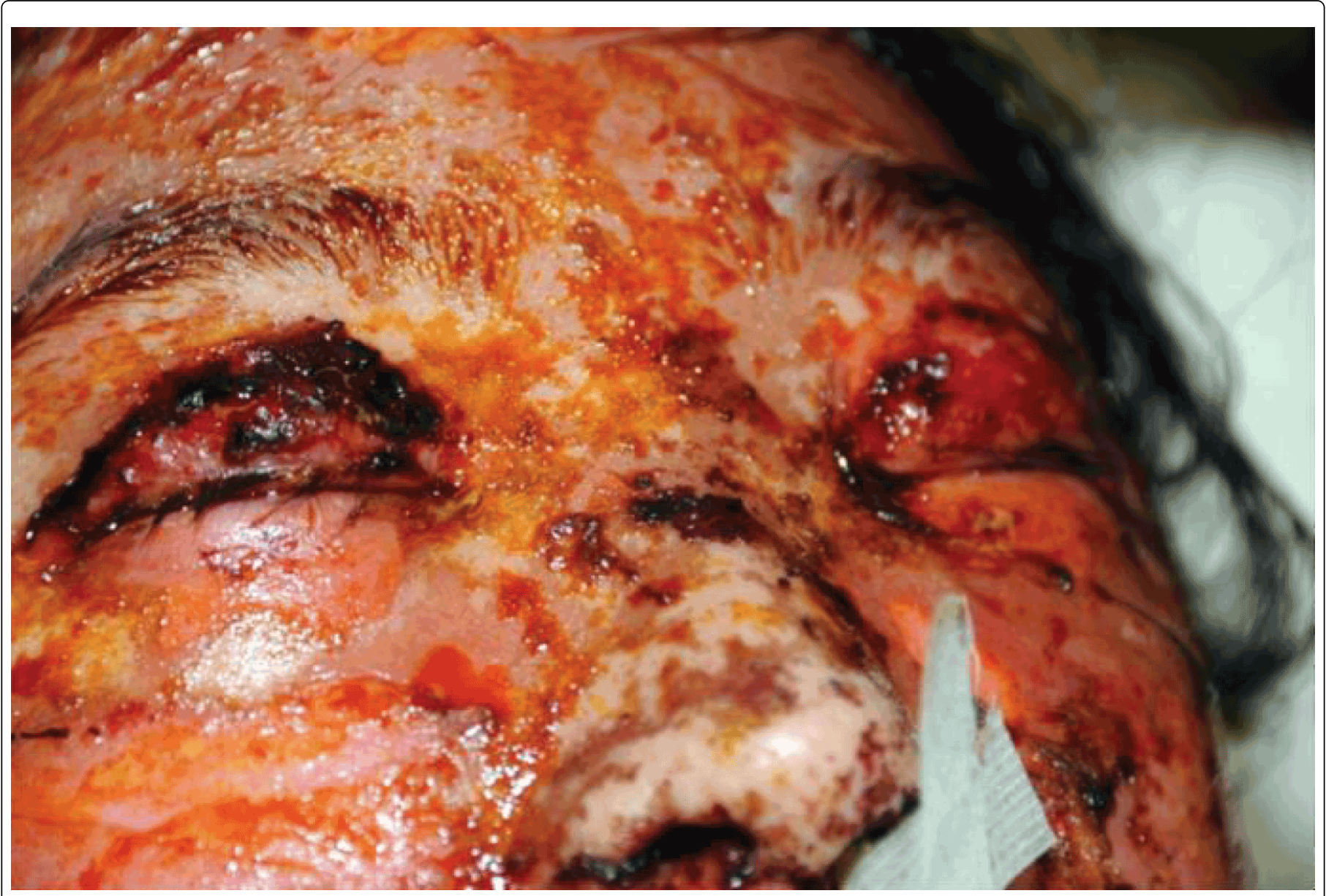

Figure 1: Appearance on Arrival at Queen Mary Hospital, Hong Kong China. Appearance showing extensive exfoliation of the epidermis.

Ophthalmological examination upon arrival showed bilateral edematous and inflamed eyelids with $1 \mathrm{~mm}$ lagophthalmos in both eyes. The conjunctiva was injected on both sides with superior and inferior symblephara as well as tarsal and forniceal ulceration. There were $80-90 \%$ corneal epithelial defects in both eyes (Figure 2).

Initial management of acute ocular surface inflammation involved daily rodding of the fornices for symblephara and adhesions, as well as intensive topical treatment to both eyes, consisting of preservative free lubricating eyedrops 1 hourly, lubricating eye ointment 2 hourly, $1 \%$ prednisolone acetate eyedrops 2 hourly and $0.5 \%$ levofloxacin eyedrops 4 times a day. Despite the above measures, the patient's ocular surface disease showed no evidence of improvement. After a thorough discussion with the pediatric intensive care physicians and the patient's family, the decision for surgical management was chosen to prevent further sequelae. The patient underwent bilateral symblepharon release and amniotic membrane transplantation under general anesthesia on $11^{\text {th }}$ of July, 10 days after disease onset. 
Citation: Shih KC, Yim SM, Chan JCY, Chan SK, Lai JSM, et al. (2014) Early Bilateral Amniotic Membrane Transplantation in the Management of Severe Ocular Involvement from Acute Toxic Epidermal Necrolysis in a Chinese Pediatric Patient. J Clin Exp Ophthalmol 5: 358. doi: $10.4172 / 2155-9570.1000358$

Page 3 of 6
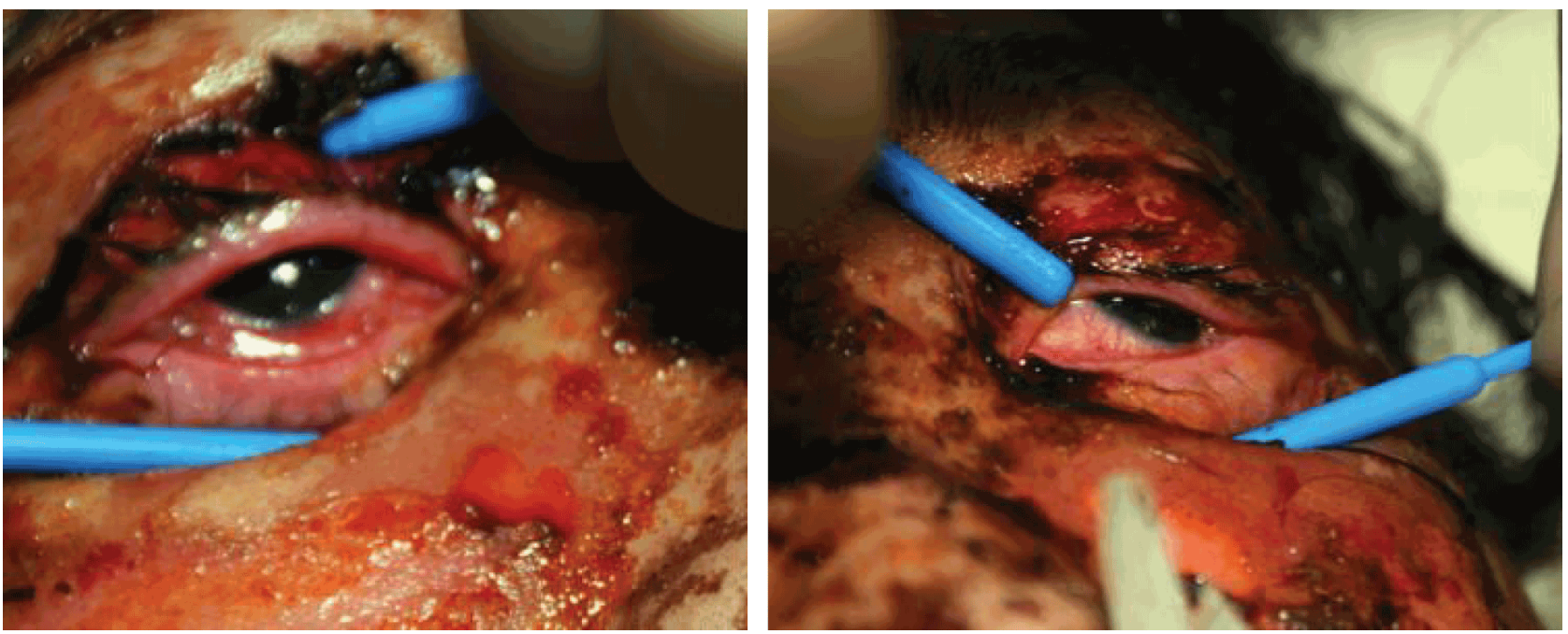

Figure 2: Intraoperative Appearance. Intraoperative appearance showing tight eyelids due to symblephara and severe ocular surface inflammation.

\section{Method}

Amniotic membrane was harvested annually under sterile conditions from a fresh placenta obtained from cesarean section at United Christian Hospital, Hong Kong. Serology from the donor and amniotic swabs were taken, ensuring no infectious agents were harbored. The AMT were then cryopreserved at $-80^{\circ} \mathrm{C}$ to provide for an immediately accessible supply. For the operation, symblepharon release and clearing of adhesive membranes was performed using a squint hook. Amniotic membranes of approximately $30 \mathrm{~mm}$ (width) $\mathrm{x}$ $40 \mathrm{~mm}$ (length) were then transplanted epithelial side up, the objective is to cover the entire cornea, bulbar conjunctiva and both upper and lower lid tarsal conjunctival surfaces. Initial anchoring sutures were performed with the edge of the AMT sutured to the anterior aspect of the upper lid margin using 8/0 vicyrl; the rest of the AMT was then draped and spread carefully onto the tarsal conjunctiva, then tucked all the way into the fornix. Three double-ended $4 / 0$ prolene sutures were placed at the upper fornix and tied outside the skin. The rest of the AMT was then draped over the bulbar conjunctiva and cornea and sutured using 10/0 nylon. A similar method was used for the lower lids and lower fornices, with the AMT end meeting with the superior AMT at mid-point, near the cornea. Postoperatively, the patient was started on $0.5 \%$ preservative free moxifloxacin eyedrops 2 hourly, $1 \%$ prednisolone acetate eyedrops 2 hourly and preservative free lubricating eyedrops 1 hourly. Rodding of the fornices was ceased after surgery.

\section{Results}

The patient was extubated with sedation weaned off on day 3 after surgery. She reported no ocular pain after regaining consciousness. During the initial 2 weeks after surgery the visual acuity was limited to hand movements in both eyes due to the intact amniotic membranes (Figure 3). By the 5th postoperative week, the amniotic membrane was resorbed and sutures were gradually removed under slit lamp guidance. Visual acuity was $6 / 18$ in the right eye and 6/12 in the left eye. There was evidence of bilateral focal point symblephera and mild punctate epithelial erosions over both corneas. However limbal palisades were preserved and extraocular movement was full for both eyes. By the $7^{\text {th }}$ postoperative week, the visual acuity was $6 / 7.5$ in the right eye and $6 / 6$ in the left eye (Figure 4). The patient eventually regained 6/6 visual acuity in both eyes by 20 weeks after surgery. Although she had bilateral residual focal symblephara and minimal keratinization of the lid margins bilaterally (Figure 5) and left nasal cornea, she experienced no ocular pain or photophobia, and was able to be weaned off topical steroids and return to normal schooling. She continues to use topical lubricants as needed for chronic dry eye of a mild grade.

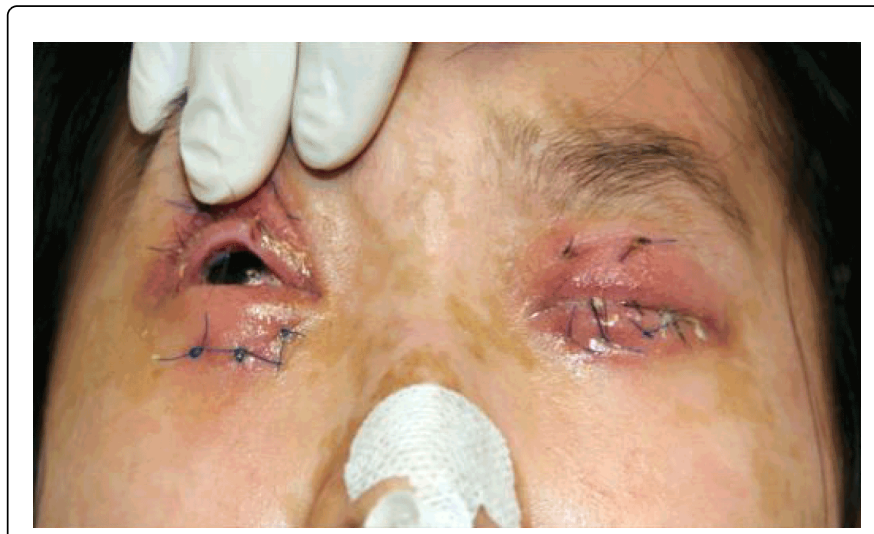

Figure 3: Post-operative Week 2. Anchoring sutures were secure and periorbital skin was healing well with erythema. There was minimal symblepharon under the AMT in the left eye. Extraocular movements were full. 
Citation: Shih KC, Yim SM, Chan JCY, Chan SK, Lai JSM, et al. (2014) Early Bilateral Amniotic Membrane Transplantation in the Management of Severe Ocular Involvement from Acute Toxic Epidermal Necrolysis in a Chinese Pediatric Patient. J Clin Exp Ophthalmol 5: 358. doi: $10.4172 / 2155-9570.1000358$

Page 4 of 6

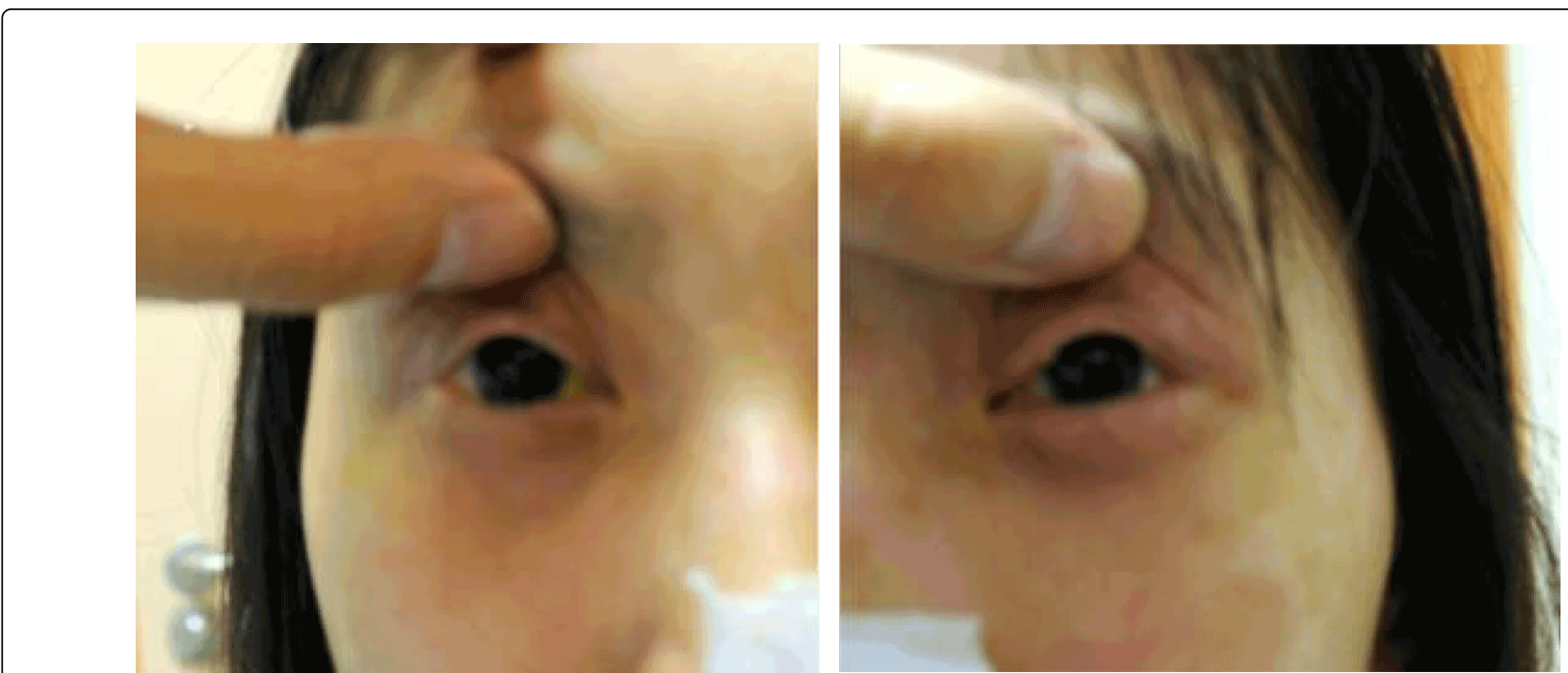

Figure 4: Post-operative Week 7. Amniotic membranes have resolved. Bilateral eyelids show residual erythema. There is keratinization of the nasal cornea. The photo on the right is of the right eye and photo on the left is of the left eye.

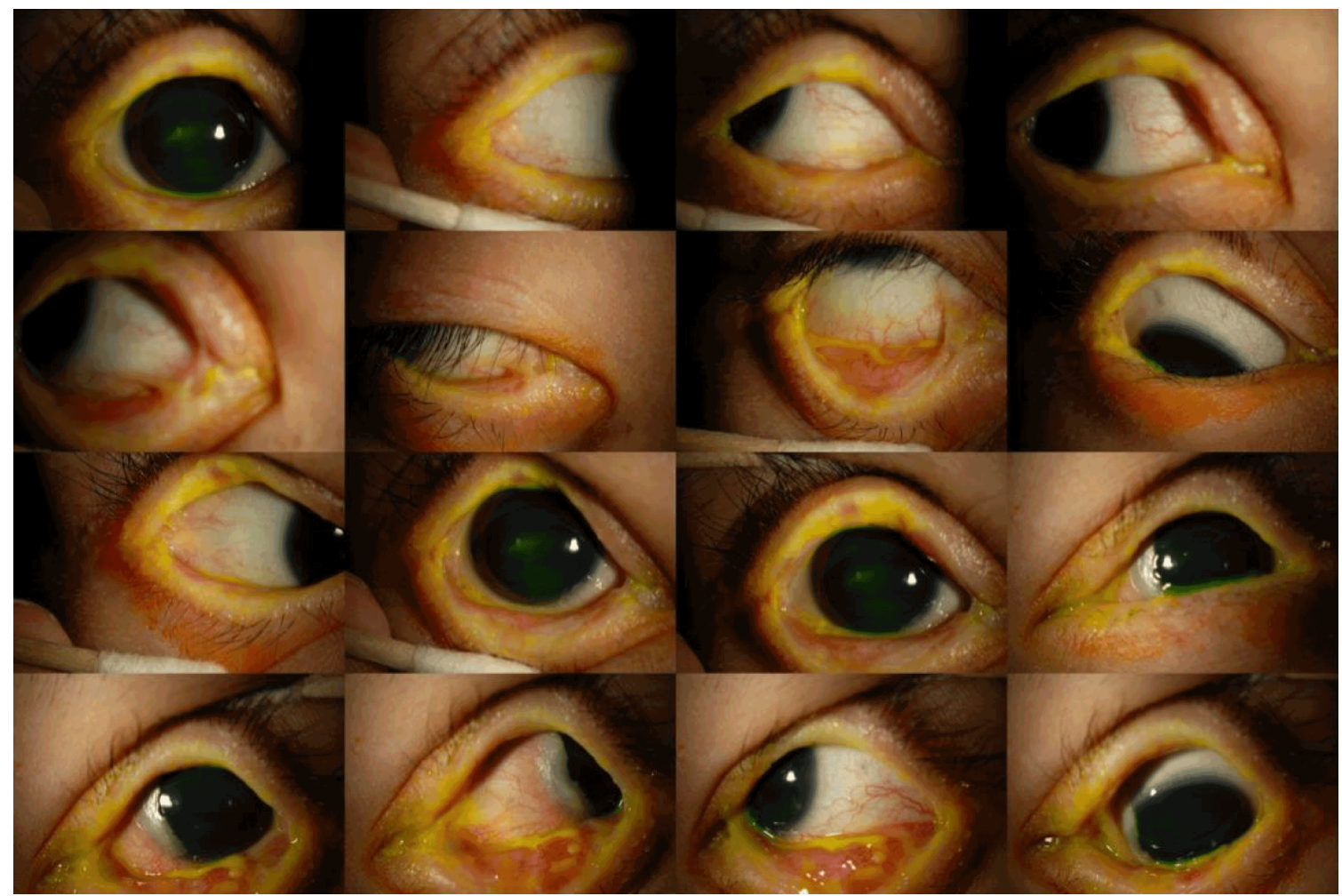

Figure 5: Post-operative Week 20. There was residual keratinization of bilateral lid margins and left nasal cornea. Extraocular movement was normal. From left to right, top to bottom photos 1-11 are of the right eye, while photos 12-16 of the left eye.

\section{Discussion}

The case report illustrates a TEN patient with good visual and structural outcome after early bilateral amniotic membrane transplantation for severe ocular surface inflammation. To our knowledge, this is the first report demonstrating the effectiveness of early AMT in a Chinese pediatric patient with TEN and adds to the growing support for early ocular intervention in patients with ocular involvement of SJS/TEN spectrum disease. 
Citation: Shih KC, Yim SM, Chan JCY, Chan SK, Lai JSM, et al. (2014) Early Bilateral Amniotic Membrane Transplantation in the Management of Severe Ocular Involvement from Acute Toxic Epidermal Necrolysis in a Chinese Pediatric Patient. J Clin Exp Ophthalmol 5: 358. doi: $10.4172 / 2155-9570.1000358$

Page 5 of 6

Ocular surface disease is a common manifestation of toxic epidermal necrolysis (TEN), with $50-67 \%$ of patients developing ocular disease, usually presenting as acute membranous or pseudomembranous conjunctivitis with sloughing of the epithelia $[14,15]$. On a cellular level, this is characterized by dermal-epidermal separation as well as epidermal apoptosis and necrosis. The underlying pathogenesis is suspected to be due to disruption of the immune system. $4-20 \%$ of patients with TEN suffer from severe ocular surface inflammation, although the severity of eye involvement does not always correspond to the severity of skin involvement or systemic illness $[3,11,16]$.

During the chronic phase of SJS/TEN, ocular surface disease is severely debilitating and is a product of conjunctival scarring, symblepharon, dry eye, conjunctivalization of cornea, corneal scar with vascularization, and eyelid malformations $[3,14,15,17,18]$. While ocular surface reconstruction in the chronic phase has been reported, it is a major surgical challenge with limited success. More promising is a paradigm shift towards disease modulation during the acute inflammatory stage. Recent literature reports have shown that early intervention with AMT in the acute phase of SJS/TEN can minimize ocular surface damage [5-13].

The amniotic membrane consists of the inner layer of the placenta. It consists of avascular cuboidal/columnar epithelia attached to a basement membrane and secretes certain growth factors, including epidermal growth factor, keratinocyte growth factor and hepatocyte growth factor, which may be useful in healing of the ocular surface [19]. When transplanted, the membrane acts as a biological bandage contact lens, providing a barrier between the denuded mucosal surfaces, thus preventing adhesion and symblephara formation. Furthermore, the amniotic membrane itself has been shown to have anti-inflammatory and anti-fibrotic effects and provides a physical scaffold for epithelial cells to migrate [20,21]. In the treatment of persistent cornea epithelial defects, there is no significant difference in re-epithelialization rates between eyes with membranes transplanted epithelial side up (overlay) or those with membranes transplanted epithelial side down (inlay) [22]. Therefore AMT can theoretically facilitate epithelialization while reducing inflammation and scarring during the acute inflammatory phase of TEN.

In the landmark report by John et al. in 2002, the authors first described the use of AMT epithelial side up (overlay) in the acute phase of TEN in four eyes of two patients suffering from severe ocular involvement: a 6-year old boy and an 8-year old girl. Furthermore the authors were the first to describe AMT over the denuded external eyelid surface and lid margin [5]. The aim of treatment was to perform AMT within 2 weeks of disease onset. To avoid amblyopia, the authors excised the membrane covering the cornea at the end of the surgery. To protect the cornea, they placed a bandage soft contact lens (BSCL) over it. Further case reports by Kobayashi et al., Atzori et al. and Tandon et al. confirmed beneficial effects of early AMT on visual and ocular outcomes [6-8]. Kobayashi et al. did not excise the membrane over the cornea, despite their patient being 6-years old. They found that the presence of the membrane did not prevent the patient from seeing and also did not prevent the medical team from performing fluorescein staining. The group also performed AMT much earlier, on day 5 of illness. Atzori et al. were the first to show that early AMT benefited elderly patients as well, with good long-term visual outcome in a 68 -year old woman.

One major limitation of AMT is that it involves a challenging surgical procedure to be performed on a gravely ill patient under general anesthesia. Alternatives include placing a Prokera device; a symblepharon ring with a sheet of amniotic membrane clipped to it, or placing the amniotic membrane unsutured over the cornea and bulbar conjunctiva. A problem of both of these alternatives is that they do not fully cover all the denuded ocular surfaces. Shammas et al. showed that in eyes which underwent Prokera or unsutured AMT alone, ocular scarring was much more severe than those that underwent sutured AMT [9]. While Prokera devices may promote epithelialization and can be done in a clinic setting under topical anesthesia, they are less effective in preventing symblephara formation and eyelid malformations. The limitations of the Prokera device in this setting was further confirmed by several case reports $[23,24]$.

The first case series on early AMT use in acute TEN was reported by Gregory in 2011. 10 consecutive patients with severe ocular involvement of SJS or TEN underwent AMT within the first 10 days of illness. All patients achieved a long-term visual acuity of $20 / 30$ or more, with $90 \%$ achieving 20/20 vision [10]. Repeat AMT every 10-14 days was helpful in those with persistent epithelial defects after initial transplantation. 2 out of 3 patients than had inferior visual outcomes had AMT done on day 10, thus the study recommended earlier treatment within the first week of illness. This was supported by a retrospective case-control series by $\mathrm{Hsu}$ et al in 2012, which also showed that the best visual outcomes were in patients who underwent AMT within 5 days of disease onset and that Prokera alone was inadequate to minimize long-term ocular cicatricial complications [11]. A recent study by Kim et al looked into 51 consecutive patients with SJS or TEN. Amongst them, patients younger than 18 years of age had significantly more severe ocular involvement. Although AMT was shown to have beneficial effects on the ocular surface, this study crucially found that early intervention with intravenous immunoglobulin (IVIG) and corticosteroids led to significantly improved long-term visual outcomes [12]. Hence good systemic treatment by a physician is just as important in the management of acute ocular inflammation.

Most recently, a prospective case series by Lopez-Garcia et al. on AMT for moderate and severe ocular involvement of TEN showed that the observed clinical improvement in ocular surface condition and visual acuity corresponded with a reduction in squamous metaplasia in corneal and conjunctival cells and increase in goblet cell density over 1 year [13]. This is the first study to demonstrate the beneficial effects of AMT were linked to histological improvements of the ocular surface.

When applying this evidence into practice, it is important to also note that there is a lack of standardization of amniotic membrane quality. There is considerable inter- and intra-donor variation in the quality of amniotic membrane. Furthermore the time from harvesting to use is different in each center, depending on availability. At our center, amniotic membrane is harvested annually and then cryopreserved at $-80^{\circ} \mathrm{C}$ for up to one year. It has been shown that the biologically active growth factors are depleted over time. Hence clinical efficacy from the mentioned studies may not be universal.

In conclusion, results from this case report add to the growing evidence supporting early AMT in minimizing ocular surface damage and preventing severe vision loss in patients acute TEN. To our knowledge this is the first case written on a Chinese pediatric patient with severe ocular involvement of acute TEN. Effective management is dependent on early and active communication between the patient's family, pediatricians, intensive care physicians and ophthalmologists. 
Citation: Shih KC, Yim SM, Chan JCY, Chan SK, Lai JSM, et al. (2014) Early Bilateral Amniotic Membrane Transplantation in the Management of Severe Ocular Involvement from Acute Toxic Epidermal Necrolysis in a Chinese Pediatric Patient. J Clin Exp Ophthalmol 5: 358. doi: $10.4172 / 2155-9570.1000358$

Page 6 of 6

\section{Special Thanks}

Gratitude is to be given to Prof. Michael Taravella and Dr. Darren Gregory for their advice on management via email regarding this case.

\section{Declaration}

Written consent has been obtained from the parents of the patient for publication of her case report in a referred journal.

All authors certify that they have NO affiliations with or involvement in any organization or entity with any financial interest (such as honoraria; educational grants; participation in speakers' bureaus; membership, employment, consultancies, stock ownership, or other equity interest; and expert testimony or patent-licensing arrangements), or non-financial interest (such as personal or professional relationships, affiliations, knowledge or beliefs) in the subject matter or materials discussed in this manuscript.

\section{References}

1. LYELL A (1956) Toxic epidermal necrolysis: an eruption resembling scalding of the skin. Br J Dermatol 68: 355-361.

2. Roujeau JC (1994) The spectrum of Stevens-Johnson syndrome and toxic epidermal necrolysis: a clinical classification. J Invest Dermatol 102: 28S-30S.

3. Yip LW, Thong BY, Lim J, Tan AW, Wong HB, et al. (2007) Ocular manifestations and complications of Stevens-Johnson syndrome and toxic epidermal necrolysis: an Asian series. Allergy 62: 527-531.

4. Honavar SG, Bansal AK, Sangwan VS, Rao GN (2000) Amniotic membrane transplantation for ocular surface reconstruction in StevensJohnson syndrome. Ophthalmology 107: 975-979.

5. John T, Foulks GN, John ME, Cheng K, Hu D (2002) Amniotic membrane in the surgical management of acute toxic epidermal necrolysis. Ophthalmology 109: 351-360.

6. Kobayashi A, Yoshita T, Sugiyama K, Miyashita K, Niida Y, et al. (2006) Amniotic membrane transplantation in acute phase of toxic epidermal necrolysis with severe corneal involvement. Ophthalmology 113: 126-132.

7. Atzori L, Peiretti E, Ferreli C, Pinna AL, Fossarello M, et al. (2006) Amniotic membrane transplantation in the surgical management of symblepharon following toxic epidermal necrolysis. J Eur Acad Dermatol Venereol 20: 214-216.

8. Tandon A, Cackett P, Mulvihill A, Fleck B (2007) Amniotic membrane grafting for conjunctival and lid surface disease in the acute phase of toxic epidermal necrolysis. Journal of American Association of Pediatric Ophthalmology and Stabismus 11: 612-613.

9. Shammas MC, Lai EC, Sarkar JS, Yang J, Starr CE, et al. (2010) Management of Acute Stevens-Johnson Syndrome and Toxic Epidermal Necrolysis Utilizing Amniotic Membrane and Topical Corticosteroids. Am J Ophthalmol 149: 203-213.
10. Gregory DG (2011) Treatment of acute Stevens-Johnson syndrome and toxic epidermal necrolysis using amniotic membrane: a review of 10 consecutive cases. Ophthalmology 118: 908-914.

11. Hsu M, Jayaram A, Verner R, Lin A, Bouchard C, (2012) Indications and Outcomes of Amniotic Membrane Transplantation in the Management of Acute Stevens-Johnson Syndrome and Toxic Epidermal Necrolysis: A Case-Control Study. Cornea 31: 1394-1402.

12. Kim KH, Park SW, Kim MK, Wee WR (2013) Effect of age and early intervention with a systemic steroid, intravenous immunoglobulin or amniotic membrane transplantation on the ocular outcomes of patients with Stevens-Johnson syndrome. Korean J Ophthalmol 27: 331-340.

13. López-García JS, Rivas L, García-Lozano I, Conesa E, Elosua I, et al. (2014) Amniotic membrane transplantation in acute toxic epidermal necrolysis: histopathologic changes and ocular surface features after 1year follow-up. Eur J Ophthalmol 24: 667-675.

14. Power WJ, Ghoraishi M, Merayo-Lloves J, Neves RA, Foster CS (1995) Analysis of the acute ophthalmic manifestations of the erythema multiforme/Stevens-Johnson syndrome/toxic epidermal necrolysis disease spectrum. Ophthalmology 102: 1669-1676.

15. Chang YS, Huang FC, Tseng SH, Hsu CK, Ho CL, et al. (2007) Erythema multiforme, Stevens-Johnson syndrome, and toxic epidermal necrolysis: acute ocular manifestations, causes, and management. Cornea 26: 123-129.

16. Gueudry J, Roujeau JC, Binaghi M, Soubrane G, Muraine M (2009) Risk factors for the development of ocular complications of Stevens-Johnson syndrome and toxic epidermal necrolysis. Arch Dermatol 145: 157-162.

17. De Rojas MV, Dart JKG, Saw VPJ (2007) The natural history of Stevens Johnson syndrome: patterns of chronic ocular disease and the role of systemic immunosuppressive therapy. Br J Ophthalmol 91:1048-1053.

18. Haber J, Hopman W, Gomez M, Cartotto R (2005) Late outcomes in adult survivors of toxic epidermal necrolysis after treatment in a burn center. J Burn Care Rehabil 26: 33-41.

19. Koizumi NJ, Inatomi TJ, Sotozono CJ, Fullwood NJ, Quantock AJ, et al. (2000) Growth factor mRNA and protein in preserved human amniotic membrane. Curr Eye Res 20: 173-177.

20. Gomes JA, Romano A, Santos MS, Dua HS (2005) Amniotic membrane use in ophthalmology. Curr Opin Ophthalmol 16: 233-240.

21. Tseng SC, Espana EM, Kawakita T, Di Pascuale MA, Li W, et al. (2004) How does amniotic membrane work? Ocul Surf 2: 177-187.

22. Letko E, Stechschulte SU, Kenyon KR, Sadeq N, Romero TR, et al. (2001) Amniotic membrane inlay and overlay grafting for corneal epithelial defects and stromal ulcers. Arch Ophthalmol 119: 659-663.

23. Shay E, Khadem JJ, Tseng SC (2010) Efficacy and limitation of sutureless amniotic membrane transplantation for acute toxic epidermal necrolysis. Cornea 29: 359-361.

24. Kolomeyer AM, Do BK, Tu Y, Chu DS (2013) Placement of ProKera in the management of ocular manifestations of acute Stevens-Johnson syndrome in an outpatient. Eye Contact Lens 39: e7-11. 